\title{
Scanning laser polarimetry of the retinal nerve fibre layer in primary open angle and capsular glaucoma
}

\author{
Gábor Holló, Ildikó Süveges, Attila Nagymihály, Péter Vargha
}

\begin{abstract}
Aims-To evaluate the clinical value of scanning laser polarimetry with the nerve fibre analyser type II in primary open angle glaucoma (POAG) and capsular glaucoma.

Methods-Scanning laser polarimetry was performed on one eye of 30 patients suffering from POAG, 25 patients suffering from capsular glaucoma, and on 35 healthy control subjects. The retinal nerve fibre layer (RNFL) thickness values were compared among the groups. Reproducibility of the measurements was calculated and the influence of pilocarpine induced miosis on the results was investigated.

Results-RNFL thickness in the superior and inferior sectors, as well as along the total circumference was significantly lower in both glaucoma groups than in the control eyes $(p<0.05)$. None of the thickness values differed between the two glaucoma groups. Reproducibility was comparable in all groups; the coefficient of variation varied between $3.0 \%$ and $8.9 \%$ for the different sectors investigated. Miosis had no significant impact either on the thickness values or on the reproducibility $(\mathrm{p}>0.05)$.

Conclusion-The results suggest that scanning laser polarimetry is a useful method for nerve fibre layer analysis in glaucoma, and that it is not influenced by the pupil size.

(Br F Ophthalmol 1997;81:857-861)
\end{abstract}

1st Department of Ophthalmology,

Semmelweis

University School of

Medicine, Budapest,

Hungary

G Holló

I Süveges

A Nagymihály

Biometry Unit, Semmelweis

University School of

Medicine, Budapest,

Hungary

P Vargha

Correspondence to: Gábor Holló, 1st

Department of

Ophthalmology, Semmelweis

University School of

Medicine, 1083 Budapest,

Tömö u 25-29, Hungary.

Accepted for publication 26 June 1997 its reproducibility and compared RNFL thickness results for healthy eyes and for eyes suffer- ing from primary open angle glaucoma and from capsular glaucoma. The impact of pilocarpine induced miosis on the thickness values and reproducibility was also investigated.

\section{Subjects and methods}

Thirty patients suffering from primary open angle glaucoma (age 59.2 (SD 15.8) years), 25 patients suffering from capsular glaucoma (age 70.5 (7.9) years), and 35 healthy control subjects (age 56.6 (18.2) years) were involved in the study after informed consent was obtained from all of them. All patients were white and randomly selected from the outpatient glaucoma unit of the department. The control subjects were recruited from the gerontology unit of the university. Patients with primary open angle glaucoma had a mean intraocular pressure before treatment of more than $21 \mathrm{~mm} \mathrm{Hg}$ (based on three to four measurements) on at least 3 different days, typical glaucomatous visual field loss $(\mathrm{MD}>2.0 \mathrm{~dB}$ and/or LV $>6.0 \mathrm{~dB}^{2}$, Octopus perimeter, G1 program) and typical glaucomatous optic nerve head damage, open chamber angle, and no sign of pseudoexfoliation. Capsular glaucoma patients had intraocular pressure greater than $21 \mathrm{~mm} \mathrm{Hg}$ before treatment, typical glaucomatous visual field loss and optic nerve head damage, pseudoexfoliative material on the iris and crystalline lens with characteristic iris atrophy and pigmentation of the open chamber angle. Healthy control subjects had intraocular pressure consistently below $22 \mathrm{~mm} \mathrm{Hg}$, optic nerve heads of normal appearance, no visual field defects and no sign of pseudoexfoliation. Also, they had no history of ophthalmic diseases. One eye per subject (randomly selected) was evaluated.

Scanning laser polarimetry was performed with a nerve fibre analyser type II (NFA II, Laser Diagnostic Technologies Inc, San Diego, CA, USA, software version 2.1.15). This noninvasive technique has been described in detail elsewhere. ${ }^{2-7}$ In brief, NFA II projects $780 \mathrm{~nm}$ polarised laser light on the retina. The birefringent, parallel nerve fibres cause a change in the polarisation of the light reflected from the eye to the detector. The degree of this change corresponds to the thickness of the RNFL, and is analysed by the built in polarisation detection unit. RNFL thickness is calculated automatically in each of $256 \times 256$ individual retinal positions. The measuring ellipse is defined by the operator between limits of 1.5 and 2.0 disc 
diameters around the disc, concentric with its margin. Mean thickness values along the measuring ellipse and their ratios are calculated by the software for the total circumference and along the superior, temporal, inferior, and nasal quadrants of the retinal image. A polarisation image is shown on the display screen (Figs 1 and 2). Baseline images can be calculated from two to eight images of the same eye using the software. 'Variance' (standard deviation divided by the mean for each pixel position, averaged for all pixels) of the images used for the calculation of the baseline image is provided by the software. It is unnecessary to define a reference plane or to correct for magnification. In order to compensate for polarisation caused by the central cornea and the crystalline lens, a compensator unit is built into the instrument. Because the compensation range is exceeded by the high polarisation caused by the peripheral cornea, axial illumination and undilated pupils are recommended.

The measurements were performed in each case by the same trained examiner (AN). An external fixating light was used, the field size was set to $15 \times 15^{\circ}$. The pupils were undilated. Patients refrained from using drops containing pilocarpine for 12 hours before the measurements. The optic nerve head was positioned in the centre of the display, and three good quality images were obtained. A few minutes later three further good quality images were obtained. In 24 glaucomatous eyes the pupil diameter was measured, then $1 \%$ pilocarpine drops were instilled. One hour later the pupil diameter was measured again and the complete image acquisition process was repeated.

The baseline images (first, second, first and second after pilocarpine) were calculated from the corresponding three images using the software supplied with the instrument. The disc margin was defined separately on each baseline image by the same examiner (GH). Thickness measurements were performed along a line at 1.5 disc diameters concentric with the disc margin. Default 90 degrees quadrant positions were applied: superior 45-135 degrees, inferior 225-315 degrees, nasal 135-225 degrees, and temporal 45-315 degrees. Mean RNFL thick- ness values (for superior, temporal, inferior, and nasal quadrants) and superior to inferior quadrant thickness ratio were used to compare the groups, evaluate the influence of miosis, calculate reproducibility, and correlate with visual field indices.

Analysis of variance (ANOVA) was used to compare the corresponding RNFL thickness values of the different groups. Paired comparisons were performed with the Duncan test. To eliminate the possible age effect on the differences between groups, covariance analysis (ANCOVA) was also performed. The paired $t$ test was applied for the comparison between the corresponding values obtained before and after pilocarpine instillation. Reproducibility was defined as intraindividual standard deviation, divided by the overall mean value (coefficient of variation). To compare the corresponding reproducibilities before and after pilocarpine instillation, the second power of the difference between the first and second baseline images was compared with the second power of the difference between the first and second baseline images obtained via miotic pupils using the paired $t$ test. $\mathrm{p}$ Values less than 0.05 were considered as significant.

\section{Results}

The quality of the images was satisfactory both in the control eyes and in primary open angle glaucoma. However, seven of the 25 eyes suffering from capsular glaucoma had to be excluded because of poor quality images. These eyes had dense nuclear cataracts.

In primary open angle and capsular glaucoma mean RNFL thickness in the superior and inferior quadrants as well as along the total circumference, was significantly lower than in the control group (Table 1, Figs 1 and 2). Thickness values belonging to the temporal and nasal quadrants did not differ among the groups (Table 1). None of the thickness results differed between the two glaucoma groups in a statistically significant manner (Table 1). As thickness values showed only a slight, nonsignificant age dependency covariance analysis and ANOVA gave identical results.

Table 1 RNFL thickness results for healthy eyes, eyes suffering from primary open angle glaucoma (POAG), and from capsular glaucoma (CG)

\begin{tabular}{|c|c|c|c|c|c|c|c|}
\hline & \multicolumn{7}{|c|}{ RNFL thickness ( $\mu m$ ) and superior to inferior thickness ratio (mean (SD)) } \\
\hline & \multicolumn{4}{|c|}{ Before pilocarpine treatment } & \multicolumn{3}{|c|}{ After pilocarpine treatment } \\
\hline & $\begin{array}{l}\text { (1) Control } \\
(n=35)\end{array}$ & $\begin{array}{l}\text { (2) } P O A G \\
(n=30)\end{array}$ & $\begin{array}{l}\text { (3) } C G \\
(n=18)\end{array}$ & $p$ Value & $\begin{array}{l}\text { (4) } P O A G \\
(n=23)\end{array}$ & $\begin{array}{l}\text { (5) } C G \\
(n=14)\end{array}$ & $p$ Value \\
\hline Total circumference & $64.71(8.11)$ & $57.88(8.59)$ & $57.25(9.22)$ & $\begin{array}{l}\mathrm{p}_{1-2}=0.003 \\
\mathrm{p}_{1-3}=0.03 \\
\mathrm{p}_{2-2}=0.81\end{array}$ & $56.60(6.79)$ & $56.04(9.55)$ & $\begin{array}{l}\mathrm{p}^{+} \mathrm{p}^{+-4}=0.807 \\
\mathrm{p}^{+}{ }_{3-5}=0.699\end{array}$ \\
\hline Superior quadrant & $77.52(11.53)$ & $63.01(10.84)$ & $61.02(12.05)$ & $\begin{array}{l}\mathrm{p}_{1-2}=0.0002 \\
\mathrm{p}_{\mathrm{1}_{-3}}=0.003 \\
\mathrm{p}_{2-3}=0.57\end{array}$ & $61.46(10.05)$ & $60.27(12.31)$ & $\begin{array}{l}\mathrm{p}^{+}{ }^{+-4}=0.980 \\
\mathrm{p}^{+}{ }_{3-5}=0.803\end{array}$ \\
\hline Temporal quadrant & $47.42(11.00)$ & $47.58(10.68)$ & $47.00(7.44)$ & $\mathrm{p}_{*}=0.98$ & $49.16(8.54)$ & $46.23(9.30)$ & $\begin{array}{l}\mathrm{p}^{+}+-4=0.354 \\
\mathrm{p}^{+}{ }_{3-5}=0.848\end{array}$ \\
\hline Inferior quadrant & $77.26(12.79)$ & $65.27(13.51)$ & $66.98(13.52)$ & $\begin{array}{l}\mathrm{p}_{1-2}=0.004 \\
\mathrm{p}_{1-3}=0.02 \\
\mathrm{p}_{2}=0.67\end{array}$ & $62.23(11.03)$ & $64.41(13.54)$ & $\begin{array}{l}\mathrm{p}^{+3-5}=0.482 \\
\mathrm{p}^{+}{ }^{+2-5}=0.097\end{array}$ \\
\hline Nasal quadrant & $54.85(7.62)$ & $54.29(7.69)$ & $53.03(9.14)$ & $\begin{array}{r}\mathrm{p}_{2-3}-0.01 \\
\mathrm{p}_{*}=0.75\end{array}$ & $53.03(6.88)$ & $52.06(8.43)$ & $\begin{array}{l}\mathrm{p}^{+}{ }_{2-4}=0.249 \\
\mathrm{p}^{+}{ }_{3-5}=0.924\end{array}$ \\
\hline $\begin{array}{l}\text { Superior to inferior } \\
\text { thickness ratio }\end{array}$ & $1.01(0.14)$ & $0.98(0.15)$ & $0.92(0.11)$ & $\mathrm{p}_{\mathrm{k}}=0.073$ & $1.00(0.15)$ & $0.94(0.11)$ & $\begin{array}{l}\mathrm{p}^{+}{ }_{2-4}=0.623 \\
\mathrm{p}^{+}{ }_{3-5}=0.080\end{array}$ \\
\hline
\end{tabular}




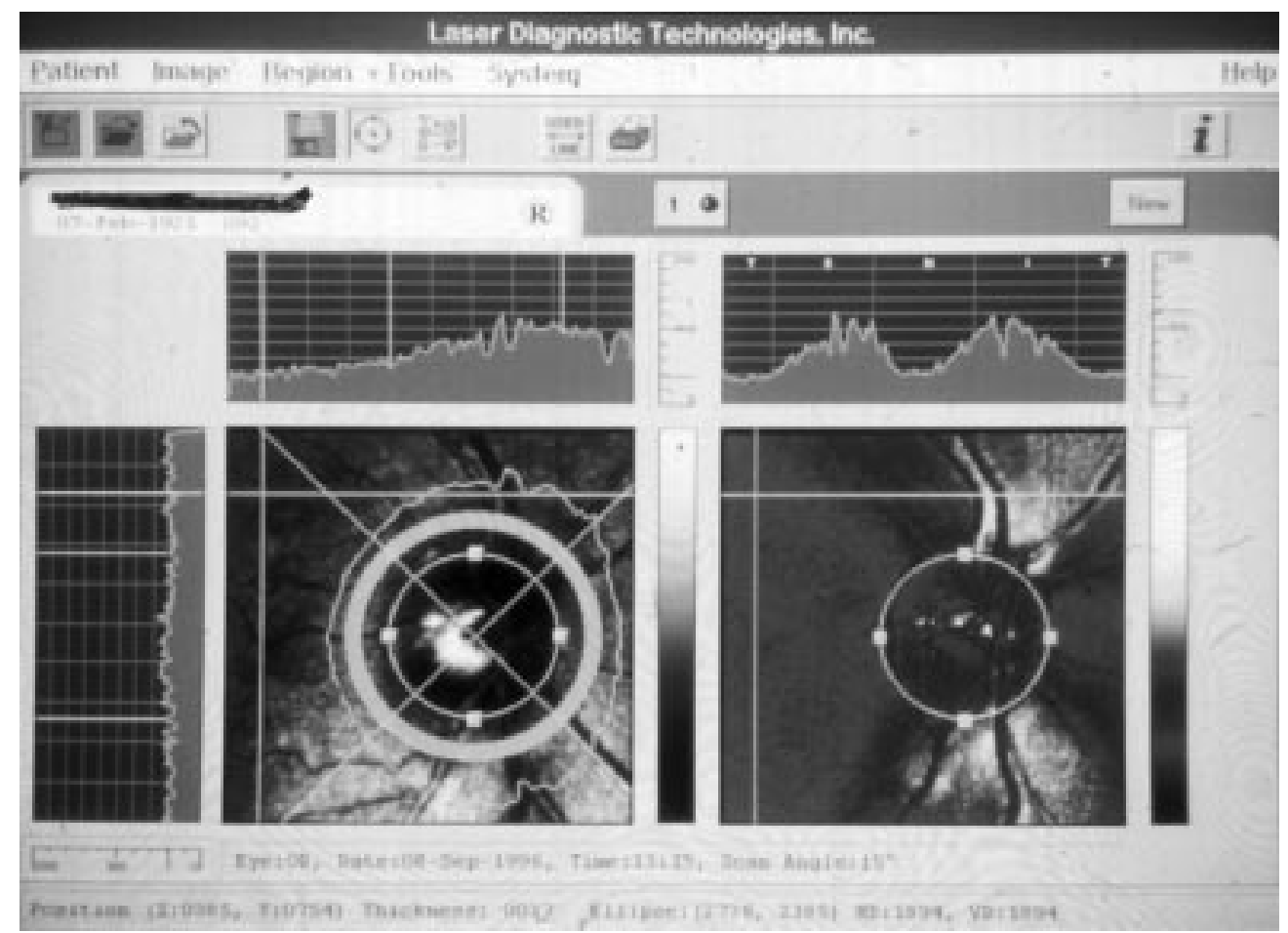

Figure 1 Scanning laser polarimetric image of a healthy eye (75-year-old female). The typical double hump of the nerve fibres is present (right image and diagram). Mean RNFL thickness values: $64.5 \mu \mathrm{m}$ for the total circumference, $78.1 \mu \mathrm{m}$ for the superior, $38.1 \mu \mathrm{m}$ for the temporal, $85.8 \mu \mathrm{m}$ for the inferior, and $54.3 \mu \mathrm{m}$ for the nasal retinal quadrant.

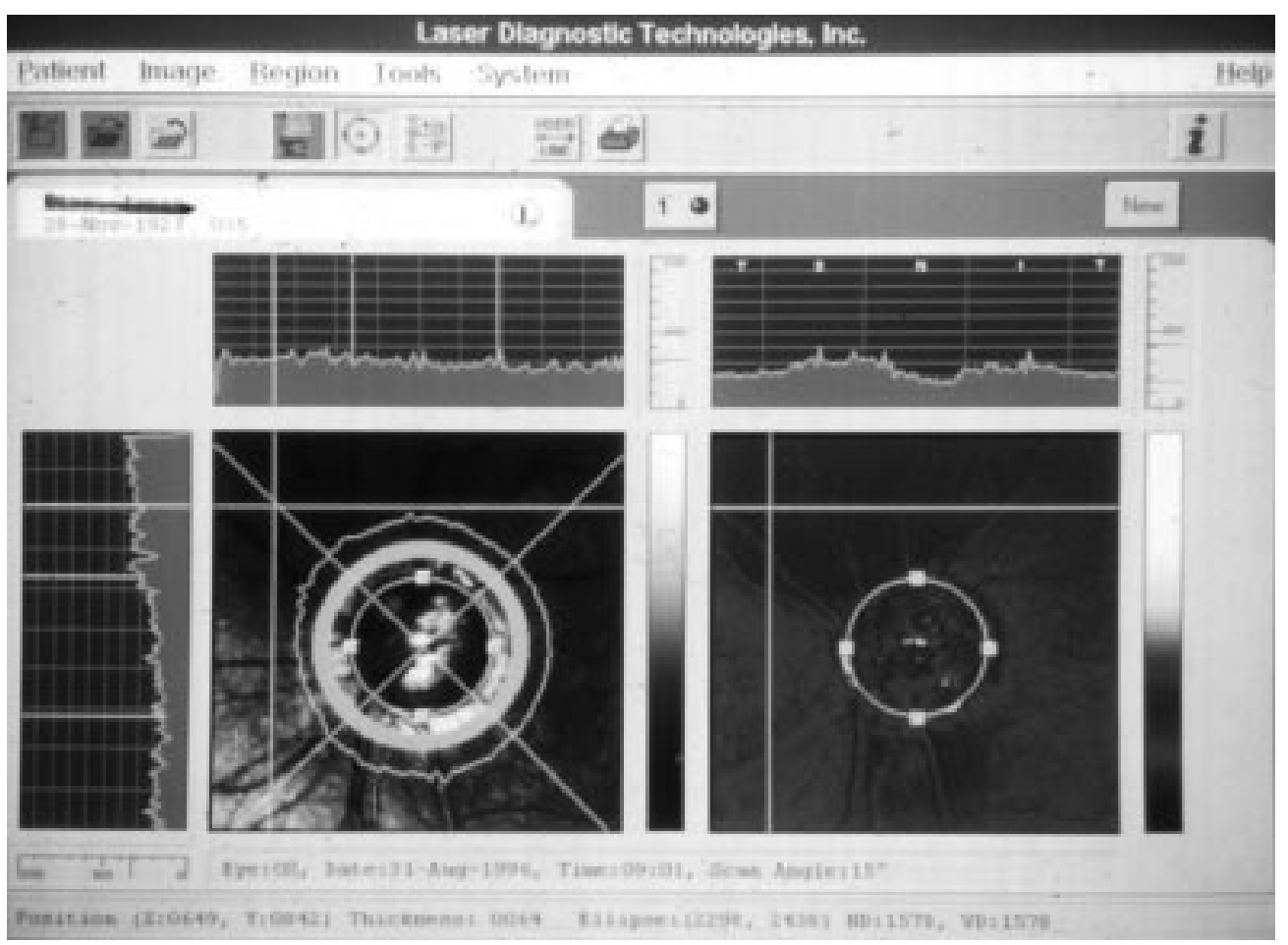

Figure 2 Scanning laser polarimetric image of an eye suffering from primary open angle glaucoma (73-year-old male). The nerve fibre loss is more advanced in the inferior quadrant, and corresponds to the notches in the neuroretinal rim left image, arrows). Mean RNFL thickness values: $51.0 \mu \mathrm{m}$ for the total circumference, $55.7 \mu \mathrm{m}$ for the superior, $45.4 \mu \mathrm{m}$ for the temporal, $55.3 \mu \mathrm{m}$ for the inferior, and $47.4 \mu \mathrm{m}$ for the nasal retinal quadrant.

The pupil diameter was 3.1 (SD 0.6) $\mathrm{mm}$ before and $1.5(0.5) \mathrm{mm}$ after pilocarpine instillation (paired $t$ test, $\mathrm{p}<0.0001$ ).

RNFL thickness values measured via miotic pupils are shown in Table 1 . The differences between the corresponding values before and after pilocarpine instillation were not statistically significant (paired $t$ test, $\mathrm{p}>0.05$ ).

Reproducibility of the measurements before and after pilocarpine treatment is shown in Table 2. The coefficients of variation were comparable in the three groups. In the control 
Table 2 Reproducibility of the measurements before and after pilocarpine instillation (paired t test)

\begin{tabular}{|c|c|c|c|c|c|c|}
\hline & \multicolumn{6}{|c|}{ Coefficient of variation (\%) of thickness and superior to inferior thickness ratio } \\
\hline & \multicolumn{3}{|c|}{ Before pilocarpine treatment } & \multicolumn{3}{|c|}{ After pilocarpine treatment } \\
\hline & $\begin{array}{l}\text { Control } \\
(n=29)\end{array}$ & $\begin{array}{l}\text { (1) } P O A G \\
(n=27)\end{array}$ & $\begin{array}{l}\text { (2) } C G \\
(n=13)\end{array}$ & $\begin{array}{l}\text { (3) } P O A G \\
(n=16)\end{array}$ & $\begin{array}{l}\text { (4) } C G \text { (4) } \\
(n=8)\end{array}$ & p Value \\
\hline Total circumference & 3.2 & 4.7 & 3.9 & 4.0 & 2.6 & $\begin{array}{l}\mathrm{p}_{1-3}=0.749 \\
\mathrm{p}_{2-4}=0.462\end{array}$ \\
\hline Superior quadrant & 4.3 & 3.7 & 4.8 & 2.9 & 4.3 & $\begin{array}{l}\mathrm{p}_{1-3}=0.604 \\
\mathrm{p}_{2-4}=0.615\end{array}$ \\
\hline Temporal quadrant & 8.5 & 8.9 & 6.1 & 8.2 & 5.2 & $\begin{array}{l}\mathrm{p}_{1-3}=0.608 \\
\mathrm{p}_{2-4}=0.824\end{array}$ \\
\hline Inferior quadrant & 4.8 & 6.3 & 3.0 & 7.3 & 3.1 & $\begin{array}{l}\mathrm{p}_{1-3}=0.268 \\
\mathrm{p}_{2-4}=0.590\end{array}$ \\
\hline Nasal quadrant & 4.7 & 7.7 & 4.8 & 5.9 & 1.7 & $\begin{array}{l}\mathrm{p}_{1-3}=0.303 \\
\mathrm{p}_{2-4}=0.218\end{array}$ \\
\hline Superior to inferior thickness ratio & 5.0 & 4.8 & 3.5 & 5.2 & 3.5 & $\begin{array}{l}\mathrm{p}_{1-3}=0.132 \\
\mathrm{p}_{2-4}=0.329\end{array}$ \\
\hline
\end{tabular}

POAG = primary open angle glaucoma, $C G=$ capsular glaucoma.

group the coefficient of variation varied between $3.2 \%$ and $8.5 \%$, in the primary open angle glaucoma group between $3.7 \%$ and $8.9 \%$, and in the capsular glaucoma group between $3.9 \%$ and $6.1 \%$. The reproducibility coefficient in miosis varied between $2.9 \%$ and $8.2 \%$ in the primary open angle glaucoma and $2.6-5.2 \%$ in the capsular glaucoma groups, respectively (Table 2). None of the coefficients of variation changed in a statistically significant manner (paired $t$ test, $\mathrm{p}>0.05$ ).

In the primary open angle glaucoma group the 'variance' of the three images used for the calculation of the baseline image (a variable of averaged reproducibility provided by the instrument) was $9.79(0.15)$ before and 11.01 (1.42) after pilocarpine instillation (paired $t$ test, $\mathrm{p}<0.005)$. In capsular glaucoma the corresponding values were 10.01 (1.69) and $10.94(1.45)$, respectively $(\mathrm{p}<0.005)$.

\section{Discussion}

Using the nerve fibre analyser type II we found scanning laser polarimetry a quick and simple technique for RNFL analysis. Evaluation of the quality of the images made it possible to exclude poor quality images unsuitable for quantitative analysis. This became important when capsular glaucoma cases were evaluated. It was impossible to obtain images of quality appropriate for analysis in seven of the 25 eyes with capsular glaucoma. The reason for the failure was the dense nuclear cataract which is frequently seen in pseudoexfoliation syndrome. ${ }^{8}$ However, in the other 18 cases image acquisition was free of difficulties, and the reproducibility of the measurement was also satisfactory.

Reproducibility was comparable in all the three groups. The coefficient of variation depended on the sector investigated, and varied between $3.0 \%$ and $8.9 \%$. Pilocarpine induced miosis caused no significant change in reproducibility. Though 'variance' between the three images used for the calculation of the baseline images increased in a statistically significant manner when the pupils became miotic, this effect cannot be considered significant clinically, since the mean increase was only 1 . On the other hand this finding corresponds to our impression that it was more difficult to obtain optimal images via small pupils.

Mean RNFL thickness values in the superior and inferior quadrants were significantly lower, both in primary open angle and in capsular glaucoma cases, than in the healthy eyes. No difference was seen, however, in the temporal and nasal values between the groups. On the display screen of the nerve fibre analyser type II the superior and inferior quadrants contain the majority of the nerve fibres in which most glaucomatous losses occur. The findings suggest that the differences detected with scanning laser polarimetry represent true glaucomatous nerve fibre losses. Though our patients suffering from capsular glaucoma were on average 14 years older than the controls, the great difference in superior and inferior RNFL thickness was not primarily caused by the age difference. It by far exceeded the calculated physiological thickness decrease, which would be only approximately $2.8 \mu \mathrm{m}$ according to the results of Chi et al ${ }^{9}$ or $5.3 \mu \mathrm{m}$ according to data from Poinoosawmy et al. ${ }^{10}$

No difference was seen between the thickness results of eyes suffering from primary open angle and capsular glaucoma, though mean age in capsular glaucoma was 11 years higher than in primary open angle glaucoma. On the other hand the age of the patients suffering from primary open angle glaucoma was very similar to the age of the healthy control subjects (59.2 (15.8) years versus 56.6 (18.2) years). Their RNFL thickness values, however, did differ significantly. These findings and the covariance analysis show that age related physiological nerve fibre loss was not the primary influence on the differences between the groups.

No statistically significant difference was found between the groups in superior to inferior thickness ratio, which is reported to be a sensitive variable for the localised RNFL loss of the individual eye. ${ }^{2}$ It would be interesting to know whether this ratio is more sensitive for differentiation when eyes with normal tension glaucoma are compared with healthy eyes or with eyes with high tension primary open angle glaucoma. 
It is of great practical importance that pilocarpine induced miosis had no significant influence on RNFL thickness results. Pilocarpine may be introduced in the treatment of the glaucomatous eye during the follow up, which might potentially interfere with the calculation of the change. It has been reported that pilocarpine induced miosis resulted in a statistically significant change of the values measured with the Heidelberg retina tomograph. ${ }^{11}$ The unchanged thickness values and reproducibility we found in miosis suggest that scanning laser polarimetry is less influenced by topical medication than optic nerve head analysis.

In summary, the results suggest that scanning laser polarimetry is a useful method for RNFL analysis in glaucoma, and in particular we found that the method is not influenced by pupil size.

The authors have no financial interest in any instrument mentioned in this paper.

1 Tuulonen A, Airaksinen J. Polarimetry of the retinal nerve fiber layer. Curr Opin Ophthalmol 1996;7:34-8.
2 Nerve Fiber Analyzer System Manual. San Diego, CA: Laser Diagnostic Technologies, Inc, 1996.

3 Anton A, Zangwill L, Emdadi A, Weinreb RN. Nerve fiber layer measurements with scanning laser polarimetry in ocular hypertension. Arch Ophthalmol 1997;115:331-4.

4 Tjon-Fo-Sang M, de Vries J, Lemij HG. Measurement by nerve fiber analyzer of retinal nerve fiber layer thickness in normal subjects and patients with ocular hypertension. $\mathrm{Am}$ formathalmol 1996;122:220-7.

5 Tjon-Fo-Sang MJ, Lemij HG. The sensitivity and specificity of nerve fiber layer measurements in glaucoma as determined with scanning laser polarimetry. Am f Ophthalmol 1997;123:62-9.

6 Weinreb RN, Shakiba S, Sample PA, Shahrokni S, Van Horn $\mathrm{S}$, Garden VS, et al. Association between quantitative nerve fiber layer measurement and visual field loss in glaucoma. Am f Ophthalmol 1995;120:732-8.

7 Weinreb RN, Shakiba S, Zangwill L. Scanning laser polarimetry to measure the nerve fiber layer of normal and glaucomatous eyes. Am f Ophthalmol 1995;119:627-36.

8 Puska P. Exfoliation syndrome: a risk factor for glaucoma and lens opacification. Helsinki: Yliopistopaino Publications, 1995:24-5.

9 Chi Q-M, Tomita G, Inazumi K, Hayakawa T, Ido T, Kitazawa Y. Evaluation of the effect of ageing on the retinal nerve fiber layer thickness using scanning laser polarimetry. f Glaucoma 1995;4:406-13.

10 Poinoosawmy D, Fontana L, Wu JX, Fitzke FW, Hitchings RA. Variation of nerve fibre layer thickness measurements with age and ethnicity by scanning laser polarimetry. $\mathrm{Br} \mathcal{F}$ Ophthalmol 1997;81:350-4.

11 Tomita G, Honbe K, Kitazawa Y. Reproducibility of measurements by laser scanning tomography in eyes before and after pilocarpine treatment. Graefes Arch Clin Exp Ophthalmol 1994;232:406-8 